Revue des sciences de l'eau

\title{
Processus d'eutrophisation : activité de la phosphatase alcaline du microplancton d'un réservoir mésotrophe marocain (Allal El Fassi) \\ Eutrophication process: alkaline phosphatase activity of microplankton in the moroccan mesotrophic reservoir (Allal El Fassi)
}

\author{
S. M. Raoui, S. Rachiq, M. Alaoui Mhamdi et N. Chadli
}

Volume 15, numéro 1, 2002

URI : https://id.erudit.org/iderudit/705435ar

DOI : https://doi.org/10.7202/705435ar

Aller au sommaire du numéro

\section{Éditeur(s)}

Université du Québec - INRS-Eau, Terre et Environnement (INRS-ETE)

ISSN

0992-7158 (imprimé)

1718-8598 (numérique)

Découvrir la revue

Citer cet article

Raoui, S. M., Rachiq, S., Alaoui Mhamdi, M. \& Chadli, N. (2002). Processus d'eutrophisation : activité de la phosphatase alcaline du microplancton d'un réservoir mésotrophe marocain (Allal El Fassi). Revue des sciences de l'eau / Journal of Water Science, 15(1), 51-62. https://doi.org/10.7202/705435ar

\section{Résumé de l'article}

L'activité de la phosphatase alcaline (APA) bactérienne et phytoplanctonique a été mesurée bimensuellement du mois de mars au mois de décembre 1998 au sein d'un réservoir mésotrophe situé dans une zone à climat semi-aride (Allal El Fassi, Maroc). Les résultats montrent que l'APA totale est importante (0,107-1,780 mmol PNP·l-1·h-1) et majoritairement d'origine bactérienne $>60$ $\%$ ) au niveau de l'épilimnion, alors qu'elle est à dominance algale (> $58 \%$ ) dans le méta- et l'hypolimnion. L'absence de corrélation entre l'APA totale et les concentrations en orthophosphates suggère que l'hydrolyse par cette enzyme n'est pas significative dans le processus de régénération du phosphore dans ce réservoir. Par conséquent, l'APA ne pourrait pas être un indicateur fiable du déficit en phosphore dans le milieu. 


\title{
Processus d'eutrophisation : activité de la phosphatase alcaline du microplancton d'un réservoir mésotrophe marocain (Allal EI Fassi)
}

\author{
Eutrophication process: alkaline phosphatase activity \\ of microplankton in the moroccan mesotrophic \\ reservoir (Allal El Fassi)
}

S.M. RAOUI ${ }^{1 *}$, S. RACHIQ ${ }^{2}$, M. ALAOUI MHAMDI ${ }^{1}$, N. CHADLI ${ }^{2}$

\section{SUMMARY}

Bacterial and phytoplanktonic alkaline phosphatase activity (APA) was measured from march to December 1998 in the mesotrophic Allal EI Fassi reservoir located in the semi-arid zone (Morocco). The total APA varied between 0.107-1.780 mmol PNP· $\mathrm{L}^{-1} \cdot \mathrm{h}^{-1}$. In epilimnion, the bacterioplankton contributed significantly $(>60 \%$ ) to total APA. In the meta and hypolimnion, the APA was predominantly algal ( $>58 \%$ ). No correlation between APA and orthophosphates indicate that the hydrolysis by this enzyme was not a significant process in recycling of phosphorus in Allal El Fassi reservoir. Consequently, the APA was not a valid test of phosphorus deficiency.

Key-words: reservoir, bacteria, algae, alkaline phosphatase, phosphorus, seasonal variations.

\section{RÉSUMÉ}

L'activité de la phosphatase alcaline (APA) bactérienne et phytoplanctonique a été mesurée bimensuellement du mois de mars au mois de décembre 1998 au sein d'un réservoir mésotrophe situé dans une zone à climat semi-aride (Allal El Fassi, Maroc). Les résultats montrent que l'APA totale est importante $\left(0,107-1,780 \mathrm{mmol} P N P \cdot l^{-1} \cdot h^{-1}\right)$ et majoritairement d'origine bactérienne $(>60 \%)$ au niveau de l'épilimnion, alors qu'elle est à dominance algale $(>58 \%$ ) dans le méta- et l'hypolimnion. L'absence de corrélation

1. Faculté sciences Dhar El Mehrez, Département de biologie, BP 1796, Fès, Maroc.

2. F.S.T. Département science de la vie, BP 2202, Fès Saïss, Maroc.

* Correspondance. E-mail: siraoui@yahoo.fr

Les commentaires seront reçus jusqu'au 31 mars 2003. 
entre l'APA totale et les concentrations en orthophosphates suggère que l'hydrolyse par cette enzyme n'est pas significative dans le processus de régénération du phosphore dans ce réservoir. Par conséquent, l'APA ne pourrait pas être un indicateur fiable du déficit en phosphore dans le milieu.

Mots clés : variations saisonnières, phosphatase alcaline, bactéries, algues, phosphore, réservoir.

\section{1 - INTRODUCTION}

La retenue de barrage Allal El Fassi est l'un des réservoirs marocains dont le bassin versant est soumis à une intense activité agricole. Une majeure partie du phosphore entrant dans le réservoir, par lessivage du sol, a conduit à l'apparition des signes d'eutrophisation précoce dans cette retenue (BOUHADDIOUl, 1997). Par ailleurs, il est admis que le phosphore est le nutriment qui contribue le plus au processus d'eutrophisation des écosystèmes aquatiques. (CAVALCANTE, 1994 ; VOLLENWEIDER, 1969). Toutefois, la forme du phosphore, que le microplancton utilise préférentiellement est le phosphore inorganique soluble $\mathrm{H}_{2} \mathrm{PO}_{4}^{-}, \mathrm{HPO}_{4}^{2-}, \mathrm{PO}_{4}^{3-}$ (CHROST et OVERBECK, 1987). En condition de déficience en phosphore, les espèces planctoniques synthétisent la phosphatase alcaline, enzyme qui hydrolyse les composés organophosphorés et libère le phosphore minéral soluble (GAGE et GORHAM, 1985 ; REICHARDT et al., 1967). De nombreux auteurs ont enregistré une corrélation inverse significative entre l'APA et les orthophosphates (BOAVIDA et HEATH, 1988 ; CHROST et al., 1984 ; HEATH et COOKE, 1975), alors que dans d'autres écosystèmes aquatiques, ces deux variables ne sont pas corrélées (BOAVIDA et MARQUE, 1995 ; JAMET et al., 1997).

Le présent travail a pour but d'étudier la part du microplancton à l'APA et la relation entre cette activité et le phosphore ( $\mathrm{P}-\mathrm{PT}, \mathrm{P}_{-} \mathrm{PO}_{4}^{3-}$ ) dans le réservoir Allal El Fassi.

\section{2 - MATÉRIEL ET MÉTHODES}

Le réservoir Allal El Fassi, lac mésotrophe, situé dans une zone semi-aride $\left(34^{\circ} \mathrm{N}, 5^{\circ} 40^{\prime} \mathrm{W}\right)$ sur l'Oued Sebou à $47 \mathrm{~km}$ de la ville de Fès (figure 1), a été mis en eau en 1992. Son volume est $84 \cdot 10^{6} \mathrm{~m}^{3}$ et sa profondeur maximale $34 \mathrm{~m}$.

Des prélèvements bimensuels d'eau ont été effectués au point le plus profond du lac, du 27 mars jusqu'au 21 décembre 1998, à l'aide d'une bouteille horizontale de type Van Dorn. Les profondeurs échantillonnées sont $0 \mathrm{~m}, 2 \mathrm{~m}$, $5 \mathrm{~m}, 10 \mathrm{~m}$, et près du fond (fond $-1 \mathrm{~m}$ ). Les paramètres abiotiques étudiés sont la température et les concentrations en $\mathrm{P}-\mathrm{PO}_{4}^{3-}$ et en $\mathrm{P}-\mathrm{PT}$. Ces dernières sont déterminées selon les méthodes standards rapportées par GOLTERMAN et al. (1978). Les abondances bactériennes sont déterminées en microscopie à 

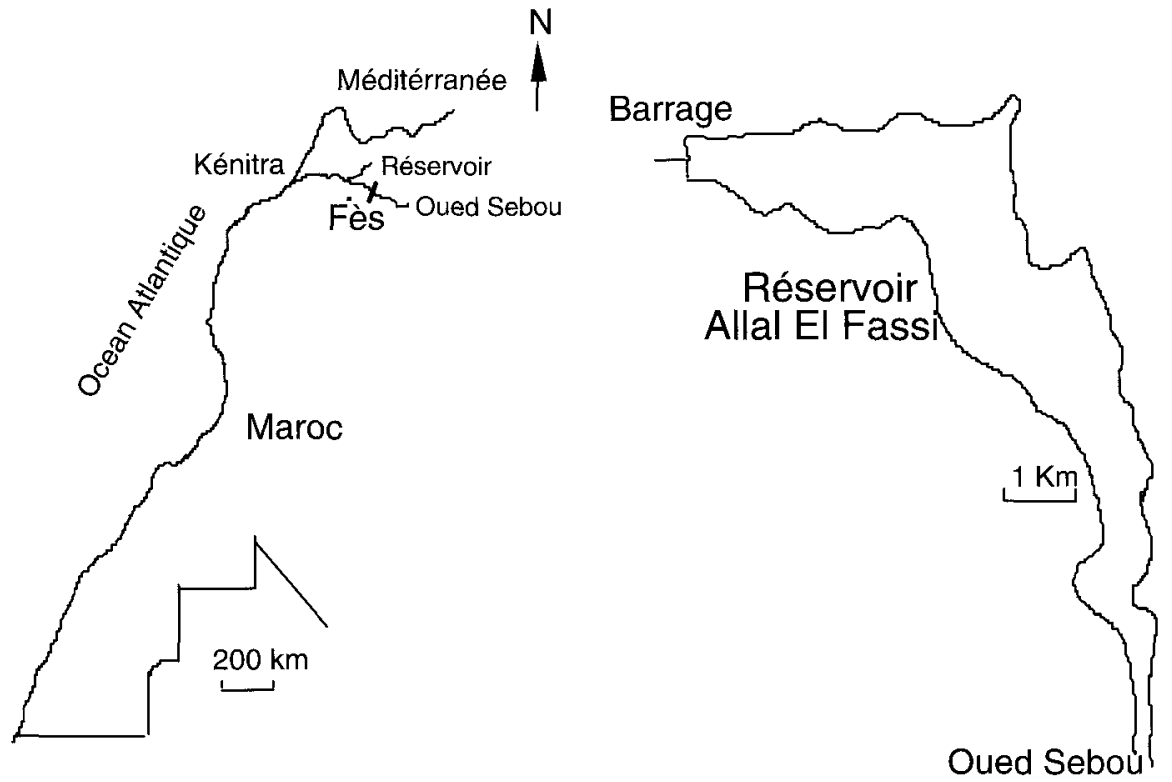

Figure 1 Localisation géographique du réservoir Allal El Fassi.

Geographic localisation of the Allal El Fassi reservoir.

épifluorescence selon le protocole proposé par HOBBIE et al. (1977), préconisant comme fluorochrome le DAPI (PORTER et FEIG, 1980). Les cellules phytoplanctoniques sont dénombrées à l'aide d'un microscope inversé (UTERMÖHL, 1958), méthode modifiée par LEGENDRE et WATT (1971). La chlorophylle a a été extraite à l'acétone $90 \%$ et dosée selon la technique rapportée par SCORUNESCO (1966).

Afin de supprimer la majeure partie de particules vivantes ou non vivantes de grande taille, l'eau brute est filtrée à travers un filet de vide de maille $100 \mu \mathrm{m}$. Le dosage de l'APA est réalisé sur des échantillons de $100 \mathrm{~mL}$ (en triplicatas), immédiatement filtrés sous vide modéré $(<100 \mathrm{~mm} \mathrm{Hg})$ à travers des membranes nucléopore de porosité $0,22 \mu \mathrm{m}$ et $0,65 \mu \mathrm{m}$. Ces filtres placés dans des tubes à hémolyse stériles sont mis en contact avec une solution ( $3 \mathrm{~mL}$ ) contenant un tampon (Tris- $\mathrm{HCl} 0,1 \mathrm{M}, \mathrm{Mg}^{++} 10^{-3} \mathrm{M}, \mathrm{pH} 8,5$ ) et $1 \mathrm{mg} \cdot \mathrm{mL}^{-1}$ de $\mathrm{p}$-nitrophényl phosphate $(\mathrm{p}-\mathrm{NPP})$. Après incubation à $37^{\circ} \mathrm{C}$ sous agitation légère pendant 6 heures, les tubes sont mis dans la glace afin d'arrêter la réaction. La lecture spectrophotométrique est réalisée à $410 \mathrm{~nm}$ (REICHARDT et al., 1967). Les résultats sont exprimés en mmol p-Nitrophénol (PNP) libéré par litre et par heure pour trois fractions de taille 0,22-100 $\mu \mathrm{m}$ (APA totale) ; 0,65-100 $\mu \mathrm{m}$ (APA liée au phytoplancton) et une fraction de 0,22$0,65 \mu \mathrm{m}$ (APA liée aux bactéries). Le dosage de l'APA libre est effectué dans la fraction dissoute après filtration des échantillons à travers un fitre de 0,22 $\mu \mathrm{m}$ de porosité. 


\section{3 - RÉSULTATS}

Le réservoir Allal El Fassi est un lac monomictique chaud (valeurs extrêmes $=12$ à $29^{\circ} \mathrm{C}$ ) présentant une stratification thermique de l'eau qui s'étend de la saison printanière jusqu'à la saison estivale (figure 2). Les concentrations en orthophosphates varient de 0 (à plusieurs reprises à $0 \mathrm{~m}$ ) et $0,2 \mathrm{mg} \mathrm{P}-\mathrm{PO}_{4}^{3-} \cdot \mathrm{L}^{-1}$ (le 15 juillet près du fond) (figure 3 ). Les variations du phosphore total suivent celles des orthophosphates et montrent des valeurs qui varient entre $0,001 \mathrm{mg}$ $\mathrm{P}-\mathrm{PT} \cdot \mathrm{L}^{-1}$ (à plusieurs reprises à $0 \mathrm{~m}$ ) et $0,69 \mathrm{mg} \mathrm{P}-\mathrm{PT} \cdot \mathrm{L}^{-1}$ (le 9 septembre près du fond) (figure 3).

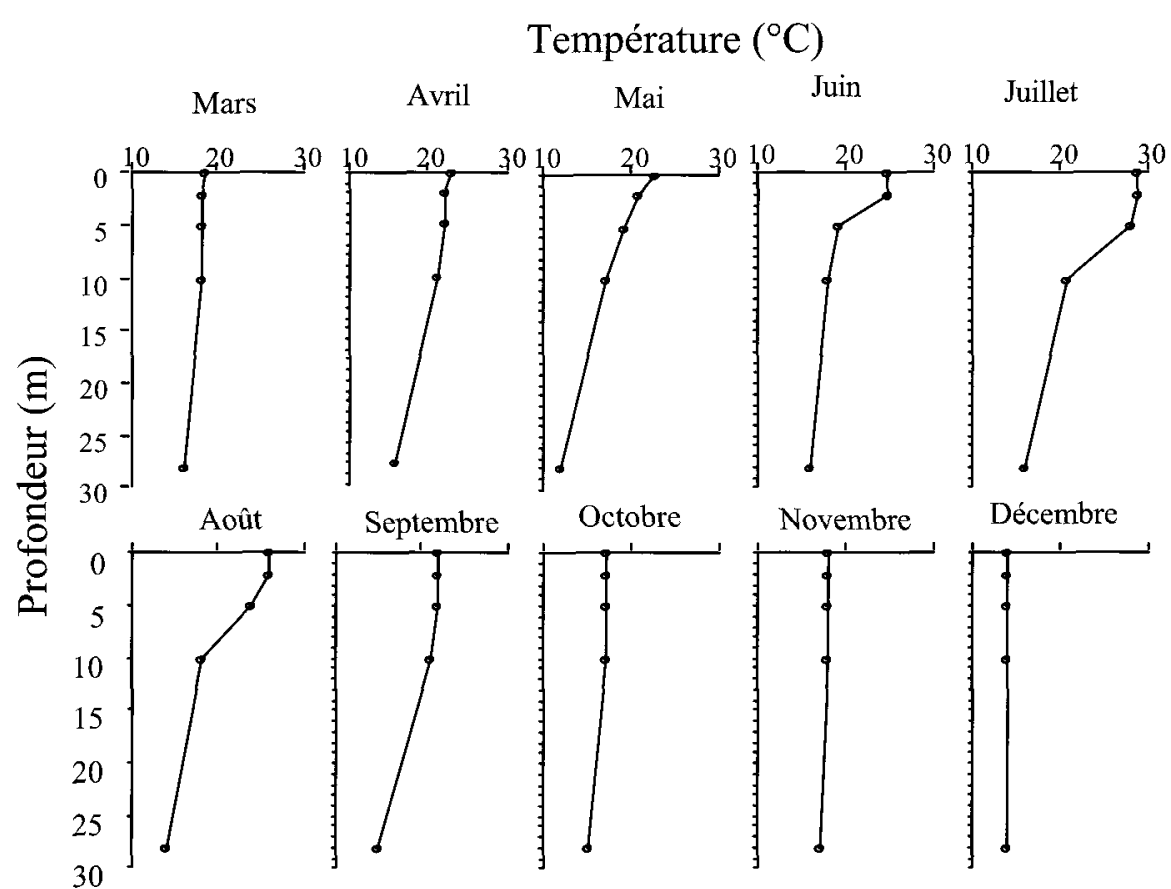

Figure 2 Variations saisonnières de la température de l'eau au niveau de la colonne d'eau.

Seasonal variations of water temperature at different depths of water column.

Les densités phytoplanctoniques varient entre $0,30 \cdot 10^{5}$ cellules $\cdot \mathrm{L}^{-1}$ (le 15 juillet près du fond) et $28 \cdot 10^{5}$ cellules $\cdot \mathrm{L}^{-1}$ (le 26 mai à $5 \mathrm{~m}$ ). La valeur moyenne sur l'ensemble de l'étude est de $6,14 \cdot 10^{5}$ cellules. $L^{-1}$ (figure 4 ). Les concentrations moyennes en chlorophylle a sont comprises entre 1,20 à $4,80 \mu \mathrm{g} \cdot \mathrm{L}^{-1}$. Pendant les périodes printanière et estivale, le phytoplancton a été dominé par les diatomées représentées par Cyclotella ocellata. Cette espèce a été accompagnée durant la saison automnale d'une population composée de Cryptomonas ovata, Peridinium cinctum, Dinobryon sertularia. Les abondances bactériennes varient entre $1,01 \cdot 10^{6}$ bactéries $\cdot \mathrm{mL}^{-1}$ (le 24 août près du fond) et $9,86 \cdot 10^{6}$ bacté- 


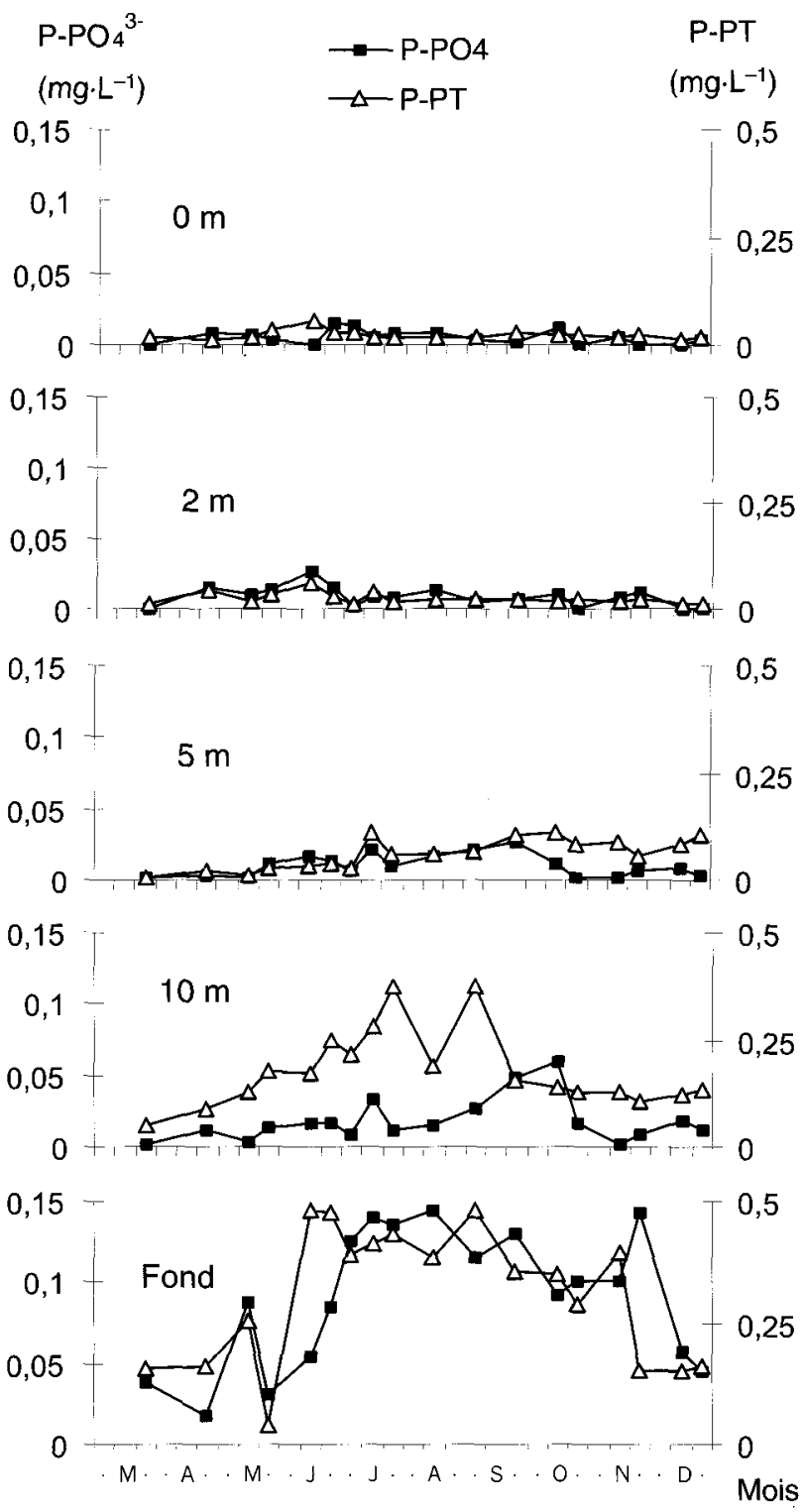

Figure 3 Variations spatiotemporelles des concentrations en orthophosphates et en phosphore total au niveau la colonne d'eau.

Spatial-temporal variations of orthophosphates and total phosphorus concentrations at different depths of water column.

ries $\cdot \mathrm{mL}^{-1}$ (le 17 avril à $10 \mathrm{~m}$ ). La moyenne sur l'ensemble des prélèvements étant de $5,34 \cdot 10^{6}$ bactéries $\cdot \mathrm{mL}^{-1}$ (figure 5 ). Ces abondances sont relativement homogènes aux niveaux $0 \mathrm{~m}, 2 \mathrm{~m}, 5 \mathrm{~m}$ et $10 \mathrm{~m}\left(5 \cdot 10^{6}\right.$ bactéries $\left.\cdot \mathrm{mL}^{-1}\right)$, alors qu'elles diminuent $\left(3,6 \cdot 10^{6}\right.$ bactéries $\left.\cdot \mathrm{mL}^{-1}\right)$ au niveau du fond du lac. 


\section{Densités algales}

$\left(\times 10^{5}\right.$ cellules $\left.\cdot L^{-1}\right)$
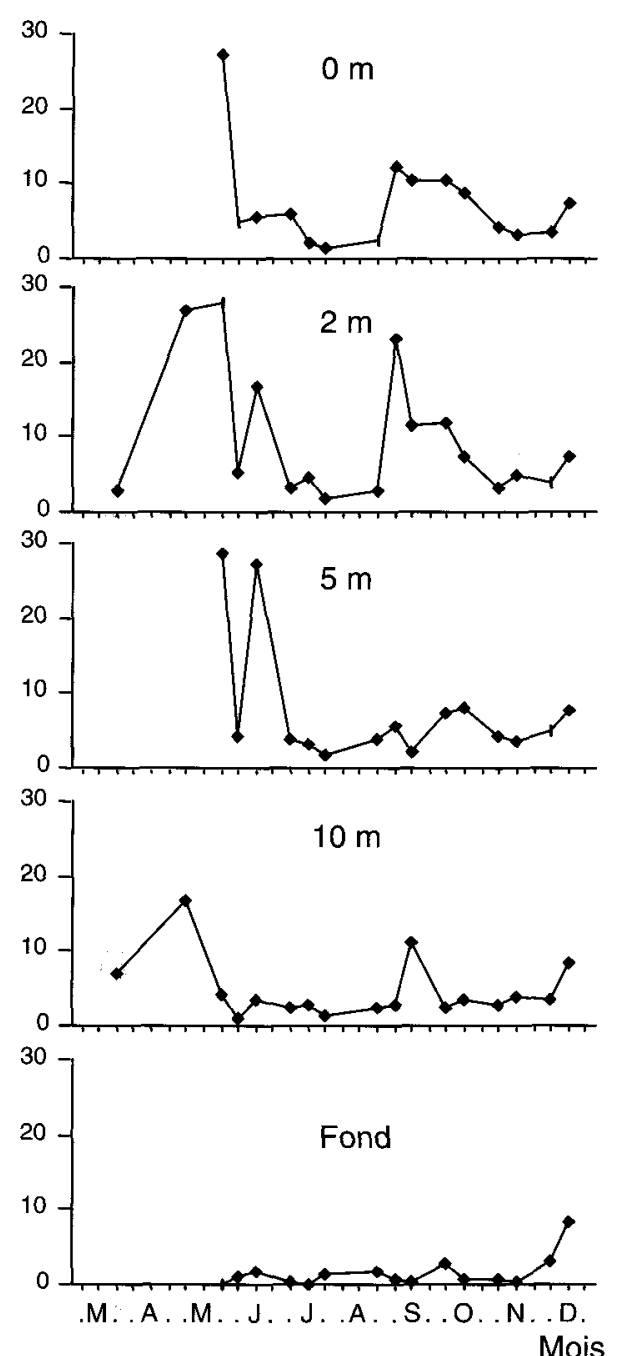

Figure 4 Variations spatiotemporelles des densités algales au niveau de la colonne d'eau.

Spatial-temporal variations of algal densities at different depths of water column.

Aucune APA liée à la fraction dissoute n'a pu être mesurée quelle que soit la profondeur considérée. L'APA totale présente des valeurs qui fluctuent entre $0,107 \mathrm{mmol} P N P \cdot L^{-1} \cdot h^{-1}$ (septembre près du fond) et $1,780 \mathrm{mmol} P N P \cdot L^{-1} \cdot h^{-1}$ (le 29 juillet à $0 \mathrm{~m}$ ) (figure 6). Les fortes activités sont enregistrées lors de la stratification thermique durant la saison estivale. L'analyse statistique par régression linéaire laisse apparaître une corrélation significative entre I'APA et 


\section{Abondances bactériennes}

$\left(\times 10^{6}\right.$ bactéries $\left.\cdot \mathrm{mL}^{-1}\right)$
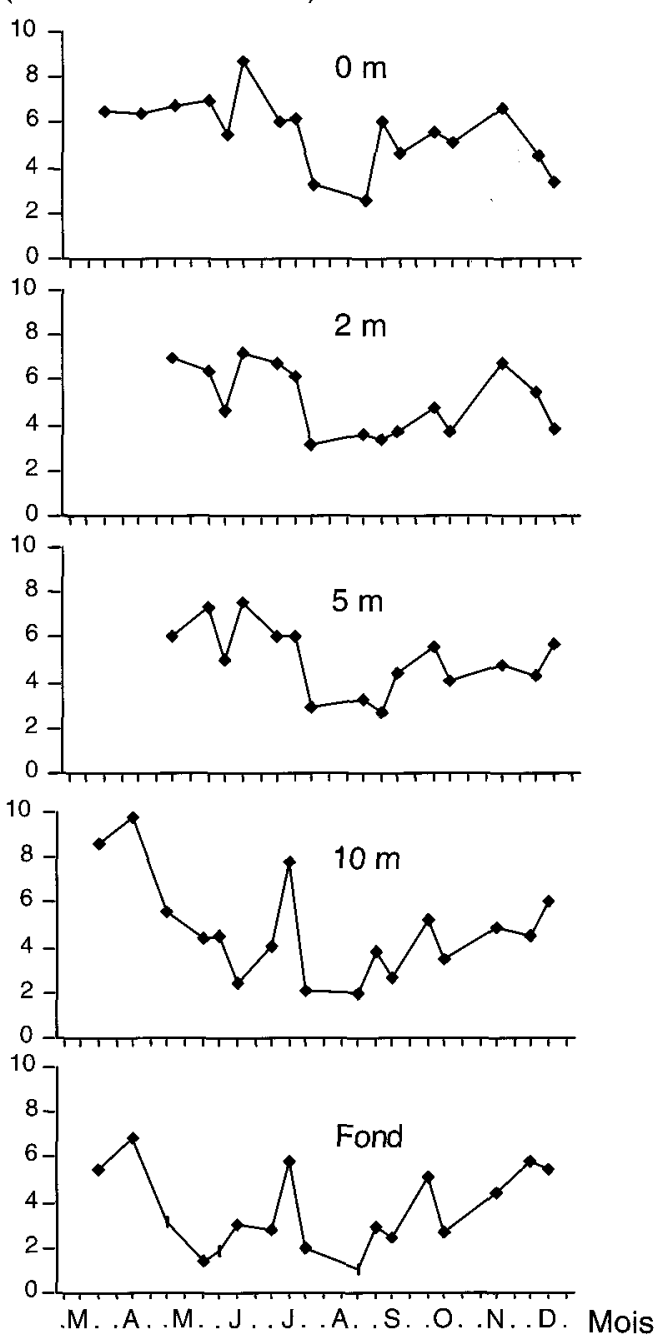

Figure 5 Variations spatiotemporelles des abondances bactériennes au niveau de la colonne d'eau.

Spatial-temporal variations of bacterial abundances at different depths of water column.

la température $(0,53<r<0,72 ; p<0,05)$ aux niveaux $0 \mathrm{~m}, 2 \mathrm{~m}$ et $5 \mathrm{~m}$. Toutefois, cette activité ne présente aucune corrélation significative $(p>0,05)$ avec les concentrations en orthophosphates et en phosphore total, le long de la colonne d'eau.

La contribution relative de la fraction de taille 0,22-0,65 $\mu \mathrm{m}$ est élevée (> $60 \%$ d'APA totale) à $0 \mathrm{~m}$ et $2 \mathrm{~m}$ (figure 7 ). À ces niveaux, l'APA de cette fraction présente une corrélation significative avec l'abondance bactérienne 


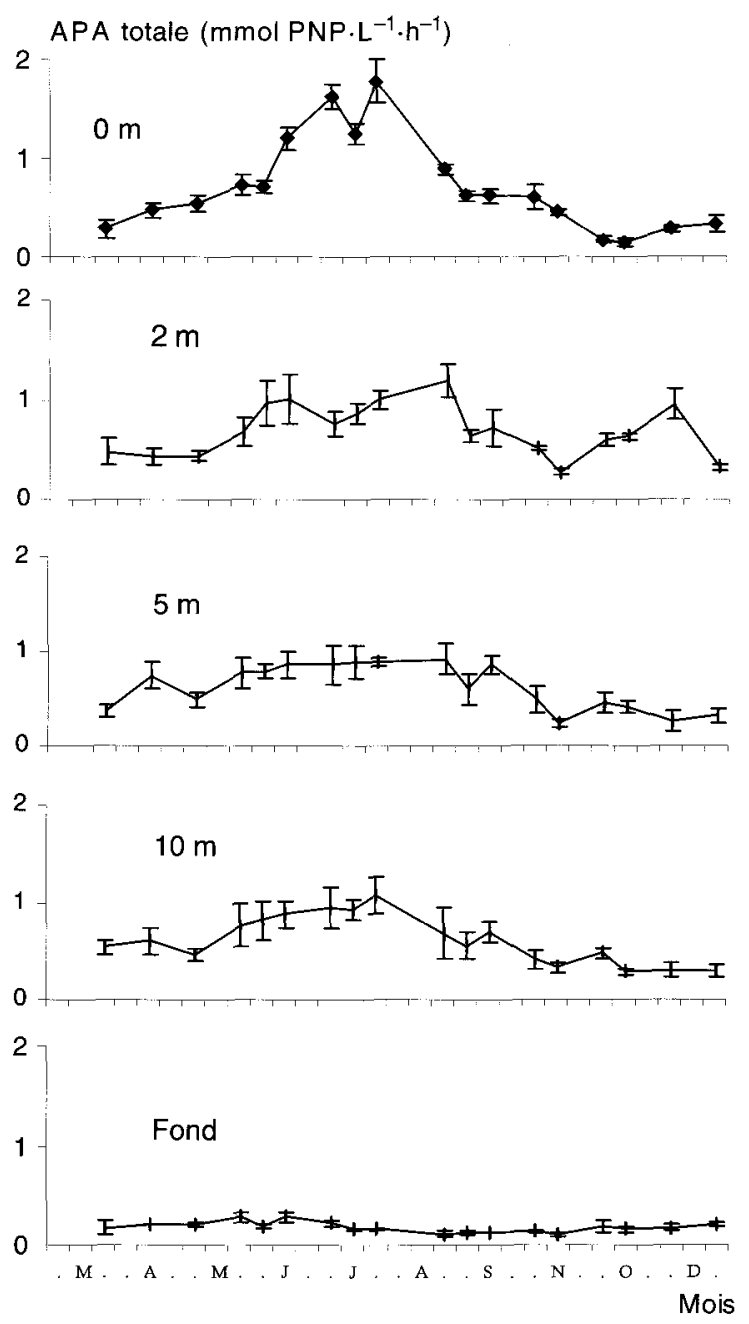

Figure 6 Variations spatiotemporelles de l'APA totale au niveau de la colonne d'eau.

Spatial-temporal variations of total APA at different depths of water column.

$(0,64<r<0,75 ; p<0,01)$. En revanche, les fortes APA liées à la fraction $0,65-$ $100 \mu \mathrm{m}(>58 \%$ d'APA totale) sont enregistrées à $5 \mathrm{~m}$ et $10 \mathrm{~m}$ (figure 7 ). À ces profondeurs, cette APA est liée par une corrélation significative à la densité algale $(0,74<r<0,80 ; p<0,05)$ et à la chlorophylle a $(0,56<r<0,62$; $\mathrm{p}<0,05)$. Ces corrélations positives témoignent sans doute d'une dépendance significative entre l'APA et le microplancton (bactéries, algues) dans ce réservoir. Toutefois, Nous notons l'absence de corrélation significative entre l'APA des deux fractions microplanctoniques et les concentrations en orthophosphates et en phosphore total le long de la colonne d'eau. 


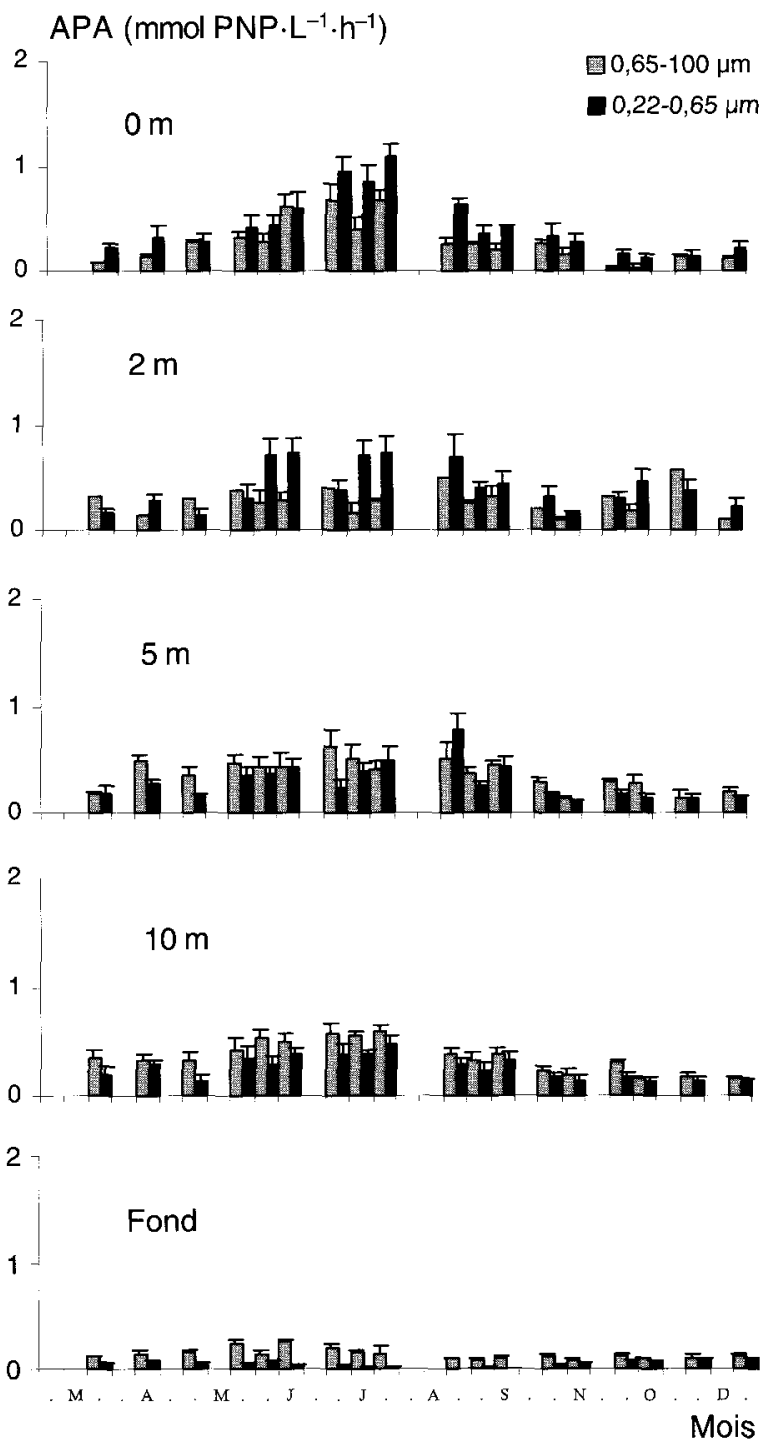

Figure 7 Contribution relative de l'APA dans les deux fractions de taille du microplancton.

Relative contribution of APA in two size fractions of microplankton.

\section{4 - DISCUSSION}

L'APA est souvent considérée comme une indication de déficience en phosphore dans le milieu aquatique (PETTERSON, 1980 ; GAGE et GORHAM, 1985). Ces auteurs ont montré l'existence de corrélation significative inverse 
entre l'APA et les concentrations en phosphore inorganique. Durant cette étude, nous notons l'absence de corrélation significative $(p>0,05)$ entre l'APA et les concentrations en orthophosphates dans toutes les fractions de taille et à toutes les profondeurs échantillonnées. Nos résultats semblent indiquer une indépendance entre l'APA et le phosphore, au moins durant la période de l'étude. Des résultats similaires ont également été observés par JAMET et al. $(1995,1997)$. De la même façon, BOAVIDA et MARQUE (1995) ont constaté l'existence d'une corrélation positive entre l'APA et les concentrations en phosphomonoesters traduisant le fait que ces derniers ne représentent pas un substrat pour cette enzyme. Par ailleurs, JANSSON et al. (1988) ont montré que les fortes APA sont enregistrées lorsque les concentrations en phosphore sont extrêmement faibles. Au niveau des couches superficielles, la chute des orthophosphates pourrait être liée probablement à une assimilation par les bactéries et/ou les algues (CAPBLANCQ, 1990) ou à leur complexation en présence d'oxygène dissous (ALAOUI et al., 1994). Au fur et à mesure que l'on s'approche des couches profondes, les concentrations en orthophosphates et en phosphore total augmentent progressivement et atteignent leurs maximums au niveau du fond. Si nos résultats indiquent que l'hydrolyse par l'APA n'est pas un mécanisme significatif dans le recyclage du phosphore, il doit y avoir probablement d'autres sources de phosphore, notamment les apports externes par le Sebou (BOUHADDIOUI, 1997), l'autolyse des bactéries et la sénescence des cellules algales (MONTIGUY et PRAIRIE, 1993) et le processus de relargage de cet élément à partir du sédiment (ALAOUl et al., 1994).

L'APA totale enregistrée pour ce réservoir $\left(0,107\right.$ à $\left.1,780 \mathrm{mmol} \cdot \mathrm{PNP} \cdot \mathrm{L}^{-1} \cdot \mathrm{h}^{-1}\right)$ excède largement celles rapportées dans différents lacs à climat tempéré (BOAVIDA et MARQUE, 1995 ; KALINOWSKA, 1997 ; JAMET et al., 1997). Les valeurs les plus élevées de cette activité ont été enregistrées au niveau des couches superficielles en période estivale lors de la stratification thermique. Cette augmentation pourrait être en relation avec les conditions environnementales du milieu. En effet, la température élevée tout au long de l'année, notamment dans l'épi- et le métalimnion, favorise sans doute une augmentation de la synthèse de cette enzyme. À l'inverse au niveau du fond, les faibles valeurs de l'APA seraient liées, d'une part, à la baisse de la température et, d'autre part, aux faibles densités bactériennes et à l'état physiologique des cellules phytoplanctoniques sénescentes.

Conformément aux résultats enregistrés par FRANCKO (1983) et ceux de JAMET et al. (1997), l'APA totale est entièrement associée aux bactéries et aux algues dans la mesure où aucune trace d'activité liée à cette enzyme n'a été révélée dans la fraction dissoute. Cette absence d'APA libre pourrait être en relation avec le niveau trophique du milieu. En effet, l'absence d'APA dans la fraction dissoute est enregistrée également dans le lac mésotrophe de Pavin (JAMET et al., 1997), alors qu'elle est généralement importante dans les milieux eutrophes et hypereutrophes (JAMET, 1995 ; KALINOWSKA, 1997).

En fonction de la profondeur, l'APA totale présente une dominance tantôt bactérienne et tantôt phytoplanctonique. Au niveau des couches superficielles, l'APA de la fraction de taille $0,22-0,65 \mu \mathrm{m}$, à majorité bactérienne, représente la majeure part d'APA totale, alors qu'elle diminue avec la profondeur au profit de celle associée à la fraction de taille 0,65-100 $\mu \mathrm{m}$, à dominance phytoplanctonique. Cette distribution est en relation avec les variations spatiotemporelles du bactérioplancton dont le maximum d'abondance est enregistré à la surface de 
la retenue. La forte contribution algale à l'APA totale pourrait être attribuée à l'apparition dans ce réservoir de populations algales dominées en grande partie par les diatomophycées représentées par C. ocellata. HINO (1988) et OLSSON (1990) ont montré que la biomasse phytoplanctonique contribue significativement à l'APA lorsqu'elle est dominée par les diatomées et les cyanobactéries.

Au terme de cette étude, l'absence de corrélation entre l'APA et les concentrations en orthophosphates suggère que l'hydrolyse par cette enzyme n'est pas significative dans le processus de régénération du phosphore dans ce réservoir. L'importance de la contribution relative des communautés bactériennes et algales à l'APA est fonction de la profondeur.

\section{REMERCIEMENTS}

Nos vifs remerciements au service régional d'hydraulique de Fès et à tout le personnel de la direction du réservoir Allal El Fassi.

\section{RÉFÉRENCES BIBLIOGRAPHIQUES}

ALAOUI MHAMDI M., ALEYA L., RACHIQ S., DEVAUX J., 1994. Étude préliminaire sur les échanges de phosphore à l'interface eau-sédiment au sein de la retenue d'Al Massira (Maroc). Rev. sci. eau, 7, 115130.

BOAVIDA M.J., HEATH R.T., 1988. Is alkaline phosphatase always important in phosphate regeneration? Arch. Hydrobiol., $111,507-518$.

BOAVIDA M.J., MARQUE R.T., 1995. Low activity of alkaline phosphatase in tow eutrophic reservoir. Hydrobiologia, 297, 11-16.

BOUHADDIOUI A., 1997. Bilan biogéochimique de l'azote et du phosphore, et dynamique des populations phytoplanctoniques de la retenue de barrage Allal El Fassi. Th. DES, FST, Fès saiss, $188 \mathrm{p}$.

CAPBLANCQ J., 1990. Nutrient dynamics and pelagic food web interaction in oligotrophic and eutrophic environments: an overview. Hydrobiologia, 207, 1-14.

CAVALCANTE P.R., 1994. Étude sur la mobilisation du phosphore, des formes azo- tées et de quelques métaux associés dans la vase eutrophe: expérimentation sur les vases de l'Erdre (in situ) et en laboratoire. Th. Doct. Univ., Nante, $164 \mathrm{p}$.

CHROST R.J., SIUDA W., HALEMEJKO G.Z., 1984. Longterm studies on alkaline phosphatase activity (APA) in lake with fish-aquaculture in relation to lake eutrophication and phosphorus cycle. Arch. Hydrobiol. Suppl., 70, 1-32.

CHROST R.J., OVERBECK J., 1987. Kinetic of alkaline phosphatase activity and phosphorus availability for phytoplankton and bacterioplankton in lake Plub see (North German eutrophic lake). Microb. Ecol., 13, 229-248.

FRANCKO D.A., 1983. Size-fractionation of alkaline phosphatase activity in lake water by membrane filtration. J. Freshwat Ecol., 2, 305-309.

GAGE M.A., GORHAM E., 1985. Alkaline phosphatase activity and cellular phosphorus as an index of the phosphorus status of phytoplankton in Minnesota lake. Freshwat. Biol., 15, 227-233. 
GOLTERMAN H.L., CLYMO R.S., OHNSTAD M.A.M., 1978. Methods for physical and chemical analyses of freshwater. IBP. Manuel $n^{\circ} 8$, ( $2^{\text {nd }}$ édition). Black. Sci. Publ., Oxford, $213 \mathrm{p}$.

HEATH R.T., COOKE G.D., 1975. The significance of alkaline phosphatase in eutrophic lake. Int. Ver. Theo. Limnol. Verh., 19, 959-965.

HINO S., 1988. Fluctuation of algal alkaline phosphatase activity and the possible mechanisms of hydrolysis of dissolved organic phosphorus in lake Barato. Hydrobiologia, 157, 77-84.

HOBBIE J.E., DALAY R.J., JASPER S., 1977. Use of nucleopore filters for counting bacteria by fluorescence microscopy. Appl. Environ. Microbiol., 33, 1225-1228.

JAMET D., 1995. Variations temporelles de l'activité de la phosphatase alkaline associée à différentes classes de taille du matériel particulaire au sein de trois lacs du massif central de degré trophiques différents. Th. Doct. Univ., Blaise Pascal, $180 \mathrm{p}$.

JAMET D., ALEYA L., DEVAUX J., 1995. Diel changes in the alkaline phosphatase activity of bacteria and phytoplankton in the hypereutrophic Villerest reservoir (Roanne, France). Hydrobiologia, 300301, 49-56.

JAMET D., AMBLARD C., DEVAUX J., 1997. Seasonal changes in alkaline phosphatase activity of bacteria and microalgea in lake Pavin (Massif Central, France). Hydrobiologia, 347, 185-195.

JANSSON M., OLSSON H., PETTERSSON K., 1988. Phosphatases: Origin, characteristics and functions in lakes. Hydrobiologia, 170, 157-175.
KALINOWSKA K., 1997. Eutrophication processes in a shallow, macrophyte dominated lake- alkaline phosphatase activity in lake Luknajno (Poland). Hydrobiologia, 342/343, 395-399.

LEGENDRE L., WATT W.D., 1971. On a rapid technique for plankton enumeration. Ann. Inst. Ocenogr., Paris, 58, 173177.

MONTIGUY C., PRAIRIE Y.T., 1993. The relative importance of biological and chemical processes in the release of phosphorus from a higly organic sediment. Hydrobiologia, 253, 141-150.

OLSSON H., 1990. Phosphatase activity in relation to phytoplankton composition and $\mathrm{pH}$ in Swedish lake. Freshwat. Biol., 23, 353-362.

PETTERSON K., 1980. Alkaline phosphatase activity and algal surplus phosphorus and phosphorus deficiency indicator in lake Erken. Arch. Hydrobiol., 89, 54-87.

PORTER K.G., FEIG Y.S., 1980. Use of DAPI for identifying and counting aquatic microflore. Limnol. Oceanogr., 25, 943948.

REICHARDT W., OVERBECK J., STEUBING L., 1967. Free dissolved enzyme in lake waters. Nature, 216, 1345-1347.

SCOR-UNESCO., 1966. Determination of phytoplankton pigments in sea water. SCOR-UNESCO., $69 \mathrm{p}$.

UTERMÖHL H., 1958. Zur vervollkommung der quantitative phytoplankton-methodik. Mitt. Int. Ver. Limnol., 9, 1-38.

VOLLENWEINDER R.A., 1969. A manual of methods measuring primary production in aquatic envirenment. IBP., Handbook 12, Blak. Sci. Publ., Oxford, 244 p. 